\title{
Conjunctival Dehiscence and Scleral Necrosis following lodine-125 Plaque Brachytherapy for Uveal Melanoma: A Report of 3 Cases
}

\author{
Duncan E. Berry ${ }^{a}$ Dilraj S. Grewal ${ }^{a}$ Prithvi Mruthyunjaya ${ }^{a}$ b \\ ${ }^{a}$ Department of Ophthalmology, Duke University Medical Center, Durham, NC, USA; ${ }^{b}$ Department of \\ Ophthalmology, Byers Eye Institute, Stanford University, Palo Alto, CA, USA
}

\section{Established Facts}

- Despite its favorable overall profile, iodine-125 brachytherapy is associated with complications, including keratitis, corneoscleral necrosis, iris neovascularization, radiation-induced cataract, neovascular glaucoma, radiation retinopathy, and optic neuropathy.

- No cases of acute conjunctival dehiscence and necrosis have been reported following iodine-125.

\section{Novel Insights}

- We present 3 cases of acute conjunctival dehiscence and scleral necrosis occurring 2-6 weeks after removal of the radioactive plaque.

- Possible etiologies include conjunctival microinfection, surgically-induced necrotizing scleritis, dehiscence due to mechanical trauma, and/or acute radiation toxicity.

\section{Keywords}

Uveal melanoma - Choroidal melanoma - Plaque

brachytherapy · Conjunctival dehiscence

\begin{abstract}
Background/Aims: Plaque brachytherapy is currently the most common treatment for uveal melanoma and has many known potential complications. Here we present 3 cases of early conjunctival and scleral necrosis following iodine-125 plaque. Methods: This study was conducted as a retrospective case series. Results: We identified 3 cases of early con-
\end{abstract}

junctival and scleral necrosis following iodine-125 plaque. All patients were managed conservatively with resolution of the necrosis. Conclusions: While delayed corneoscleral necrosis following plaque brachytherapy has been previously reported, occurring many months to years after treatment, the 3 cases in this series presented within 2-6 weeks in the postoperative period. While we were unable to identify a specific etiology, we believe this represents a distinct clinical entity of post-brachytherapy cornea-scleral necrosis that is important to recognize. Possible causes include acute radiation toxicity, mechanical trauma, and/or conjunctival microinfection.

\section{KARGER}

(c) 2018 S. Karger AG, Basel

E-Mail karger@karger.com

www.karger.com/oop
Prithvi Mruthyunjaya, MD

Department of Ophthalmology

Byers Eye Institute, Stanford University Medical Center

Palo Alto, CA 94303 (USA)

E-Mail prithvi9@stanford.edu 
Table 1. Dosimetric data for each case

\begin{tabular}{llllll}
\hline Case & Plaque size & $\begin{array}{l}\text { Tumor } \\
\text { apex, } \\
\mathrm{mm}\end{array}$ & $\begin{array}{l}\text { Delivered dose } \\
\text { to tumor apex, } \\
\text { Gy }\end{array}$ & $\begin{array}{l}\text { Delivered dose } \\
\text { to outer sclera, } \\
\text { Gy }\end{array}$ & $\begin{array}{l}\text { Delivered dose } \\
\text { to inner sclera, } \\
\text { Gy }\end{array}$ \\
\hline Case 1 & $18 \mathrm{~mm}$ circular & 6 & 70.6 & 324 & 229 \\
Case 2 & $18 \mathrm{~mm}$ & 2.8 & 70.8 & 210 & 149 \\
Case 3 & $18 \mathrm{~mm}$ & 4.5 & 80.6 & 328 & 221 \\
\hline
\end{tabular}

Radiation-induced focal scleral necrosis was first reported by Jones and Reese [1] following concentrated localized gamma or beta radiation for the treatment of ocular surface squamous cell carcinomas. Scleral necrosis is an uncommon radiation complication associated with placement and removal of plaque brachytherapy because of the radioresistant nature (tolerance 15-100 Gy) of this avascular, hypocellular tissue [2]. While the theoretical risk of acute conjunctival necrosis following plaque brachytherapy has been suggested, it has not been reported to date [3].

Here we present 3 cases of conjunctival dehiscence and necrosis following iodine- 125 plaque brachytherapy. All three patients were found to have choroidal melanoma requiring treatment with iodine- 125 plaque brachytherapy with concurrent transscleral biopsy. At our institution, these patients undergo placement of the plaque in the operating room, are admitted for an inpatient stay with installation of topical antibiotics and steroids, and have the plaque removed approximately $120 \mathrm{~h}$ later. Our radiation planning procedure at that time was a based on the American Association of Physicists in Medicine (AAPM) Task Group Number 43 (TG-43) guidelines using Eclipse BrachyVision $^{\mathrm{TM}}$ (Varian Medical Systems, Inc. Palo Alto, CA, USA) as a secondary check [4]. There was no correction done for attenuation from the Silastic insert or any effects of the gold plaque - all calculations were performed assuming a homogeneous medium. Planning was calculated to the actual tumor apex even if that apex was smaller than $5 \mathrm{~mm}$. All 3 of these patients received between 70 and $80 \mathrm{~Gy}$ of radiation delivered to the apex of the tumor. Actual delivered dosing information is shown in Table 1.

The surgical technique was similar for all patients - exceptions are noted in the individual case summaries. A 360-degree conjunctival peritomy was performed followed by blunt dissection to separate the Tenon capsule. Next, each of the four rectus muscles were identified with a muscle hook, cleaned, and looped with 2-0 silk ligature. The globe was examined in each case and there were no signs of scleral thinning. All patients required disinsertion of at least one extraocular muscle which was secured in a hang-back fashion and reinserted upon plaque removal - the specific muscle is noted in the individual case summaries. Transpupillary transillumination was then performed and the tumor was identified by its casting a shadow on the transillumination. This area was then marked with blue dye ink externally. A dummy plaque was then positioned over the previously marked tumor and secured with dacron sutures. Transillumination was used to confirm adequate coverage and centration of the dummy plaque. The dummy plaque was then removed but the dacron sutures were left in place. The transscleral biopsy was performed in the bed of the previously marked tumor prior to plaque placement with the following technique. A \#57 blade was used to fashion a partial thickness scleral flap approximately $2 \times 2 \mathrm{~mm}$ in size. Next, a 27 -gauge needle attached to extension tubing was entered into the substance of the tumor and aspiration was engaged. The needle was then removed from the eye and the specimens were sent. The scleral flap was closed with 9-0 nylon suture. The plaques were secured to the sclera using the previously placed dacron sutures.

At the end of the procedure to remove the plaque, all patients received the following: (1) an intravitreal injection of bevacizumab 1.25 or $2.5 \mathrm{mg}$ given through the pars plana at a site 180 degrees from the tumor, (2) a subTenon's injection of triamcinolone acetonide $40 \mathrm{mg}$, and (3) subconjunctival injections of cefazolin and dexamethasone. Complete conjunctival closure was performed with 6-0 plain gut. Following removal of the plaque, all patients were placed on a postoperative regimen comprised of a tapering course of prednisolone acetate, ciprofloxacin, and homatropine. Any differences in management are noted in the case summaries.

\section{Case Report}

Case 1

A 31-year-old Caucasian male underwent iodine- 125 plaque brachytherapy for amelanotic choroidal melanoma. Exam revealed an amelanotic choroidal lesion with largest basal diameter (LBD) of $12.5 \mathrm{~mm}$ and a height of $5.8 \mathrm{~mm}$ located $5.0 \mathrm{~mm}$ nasal 

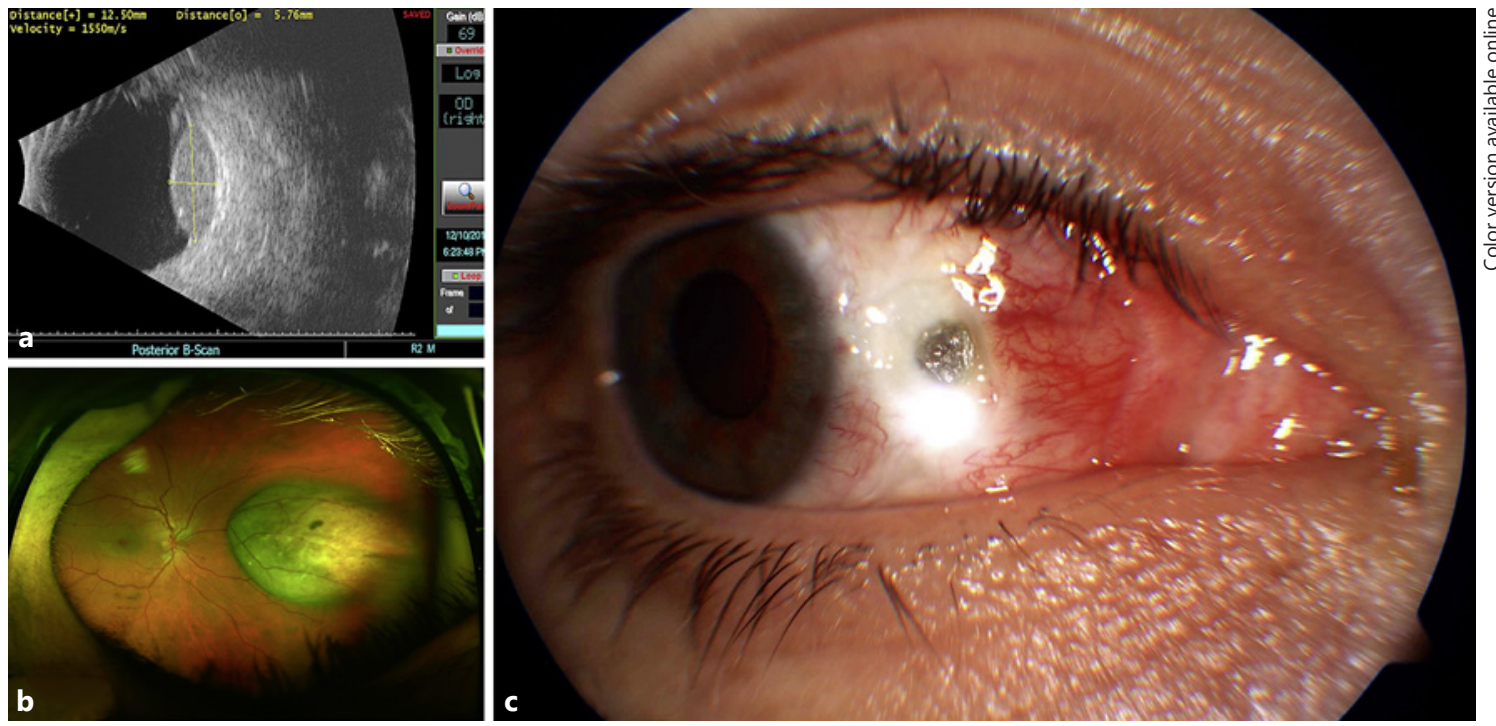

Fig. 1. a Preoperative B-scan ultrasound of the lesion. b Preoperative wide-field fundus photo demonstrating an amelanotic choroidal lesion nasal to the optic nerve in the right eye. c Postoperative week 2 visit with conjunctival dehiscence anterior to the medial rectus insertion with associated scleral thinning.

to the optic nerve in the right eye (Fig. 1a, b). He had no significant past ocular or medical history. Of note, the medial rectus was disinserted and secured in a hang-back fashion for plaque placement and then reinserted upon plaque removal. No complications were noted with regard to the disinsertion/reinsertion of this muscle.

At the 2-week postoperative visit, he was noted to have conjunctival retraction just anterior to the medial rectus insertion with associated scleral thinning (Fig. 1c). He was continued on prednisolone acetate $1 \%$ two times daily and restarted on ciprofloxacin $0.3 \%$ four times daily. This regimen was continued for 2 weeks, at which time the conjunctiva remained retracted but the area of previously exposed sclera was re-epithelialized and the ciprofloxacin was stopped. The prednisolone was continued at twice a day for the next 3 months and was then tapered. A topical nonsteroidal anti-inflammatory, bromfenac, was started at two times daily for 1 year. At last follow-up, 26 months from the date of plaque placement, no progression of scleral thinning or necrosis was noted.

Case 2

A 58-year-old Caucasian female underwent iodine- 125 plaque brachytherapy for choroidal melanoma with LBD of $14.5 \mathrm{~mm}$ and height of $2.8 \mathrm{~mm}$ with the majority of the tumor located anterior to the equator temporally in the left eye (Fig 2a, b). She had no significant past ocular or medical history. The lateral rectus and inferior oblique muscles were disinserted and secured in a hang-back fashion for plaque placement and then reinserted upon plaque removal. No complications were noted with regard to the disinsertion/reinsertion of these muscles.

At the 4-week postoperative visit, she was noted to have conjunctival wound dehiscence with exposure of vicryl suture material. The prednisolone was increased to four times daily and she was started on moxifloxacin $0.5 \%$ four times daily. This regimen was continued for 2 weeks, at which time the conjunctiva was noted to be closed and healed well without retraction or scleral exposure (Fig. 2c). The prednisolone was slowly tapered over the course of 6 weeks and was eventually discontinued. She was continued on artificial tears and has not had a recurrence to date. At last followup, 22 months from the date of the plaque placement, no progression of scleral thinning or necrosis was noted.

\section{Case 3}

Patient 3 is a 58 -year-old Caucasian female treated with iodine-125 plaque brachytherapy for amelanotic choroidal melanoma with LBD of $13.0 \mathrm{~mm}$ and height of $4.5 \mathrm{~mm}$ with the anterior border coming to the edge of pars plana and the majority of the tumor located anterior to the equator inferotemporally in the right eye (Fig 3a, b). She had no significant past ocular or medical history. The lateral rectus and inferior oblique muscles were disinserted and secured in a hang-back fashion for plaque placement and then reinserted upon plaque removal. No complications were noted with regard to the disinsertion/reinsertion of these muscles.

At the postoperative week 6 visit, she was noted to have a temporal area of avascular, elevated conjunctiva in the area of the plaque placement. Prednisolone was tapered to once daily and she was started on erythromycin ointment once daily. One week later, the area appeared to be worse with severe pain, a $6 \times 6 \mathrm{~mm}$ area of conjunctival fluorescein staining, and nodular scleritis without scleral thinning (Fig. 3c). No culture was performed at that time. She was empirically started on ciprofloxacin $0.3 \%$ four times daily, the erythromycin was increased to four times daily, and the prednisolone was stopped.

She was continued on this regimen for the next 12 weeks, during which time the original conjunctival defect slowly re-epithelialized and ultimately healed. While the conjunctival dehiscence 

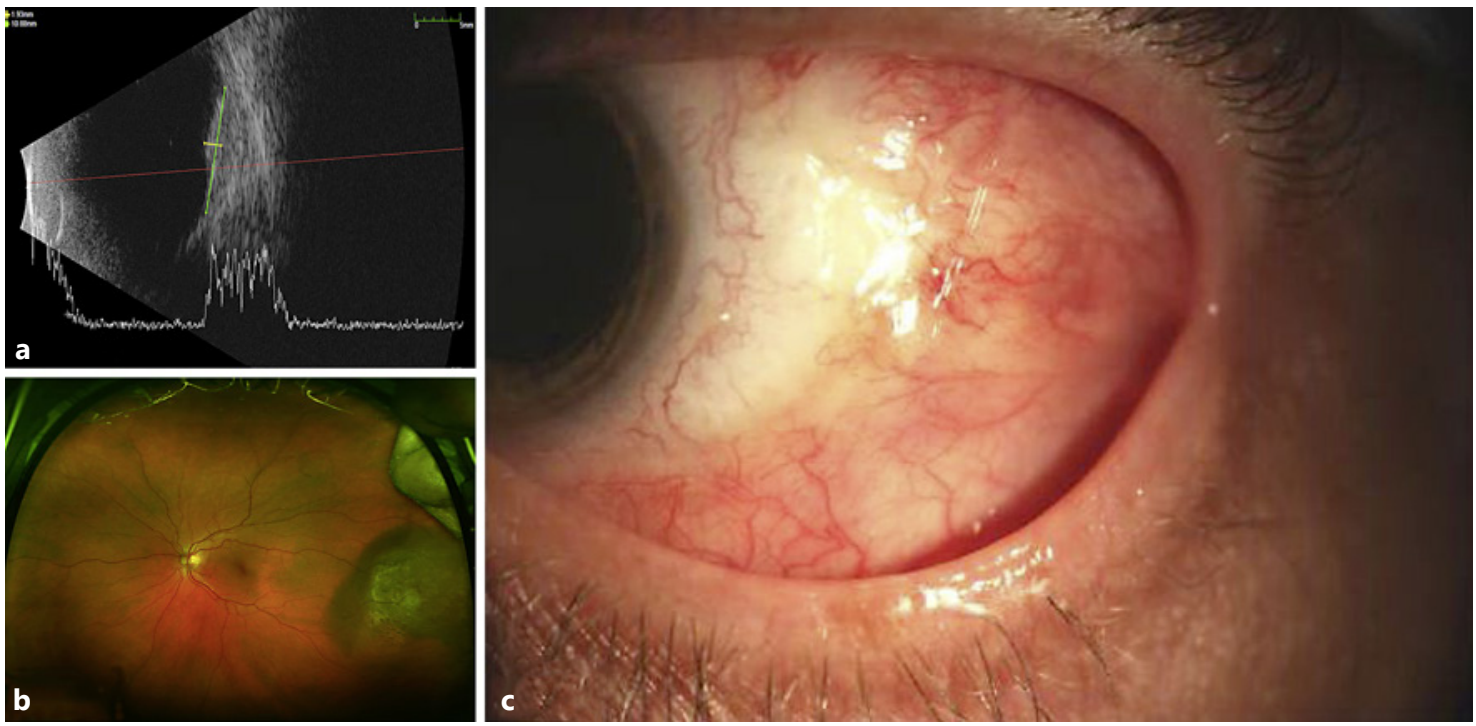

b

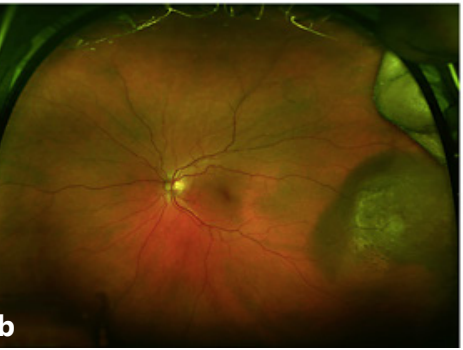

Fig. 2. a Preoperative B-scan ultrasound of the lesion. b Preoperative wide-field fundus photo demonstrating a temporal lesion in the left eye with the majority of the tumor located anterior to the equator. c Final follow-up of case 2 demonstrating a healed area of dehiscence without residual exposure.
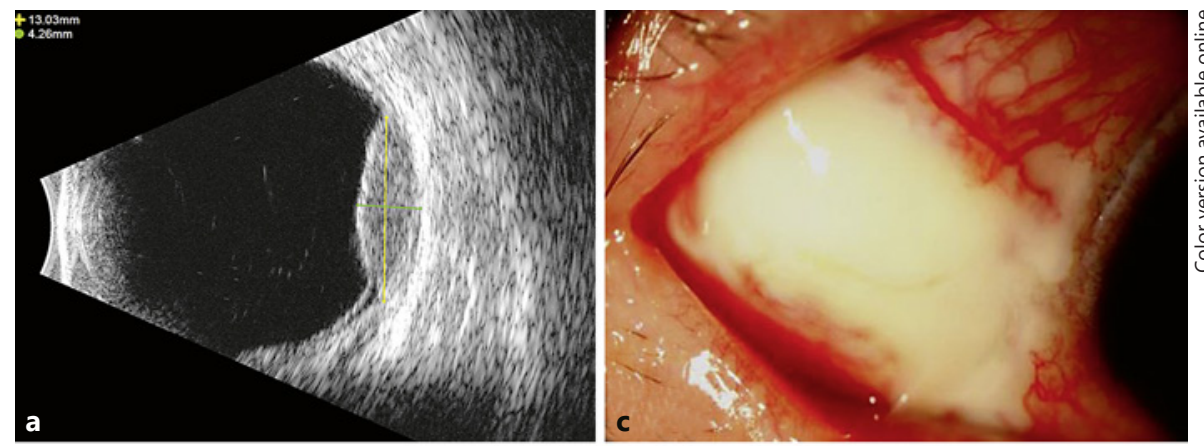

Fig. 3. a Preoperative B-scan ultrasound of the lesion. b Preoperative fundus photo (wide-field not available) demonstrating an amelanotic choroidal melanoma located inferotemporally in the right eye with the majority of the tumor located anterior to the equator. c Case 3 demonstrating conjunctival dehiscence with underlying nodular scleritis without scleral thinning in the area of the plaque placement. d Subsequent symblepharon formation following treatment.
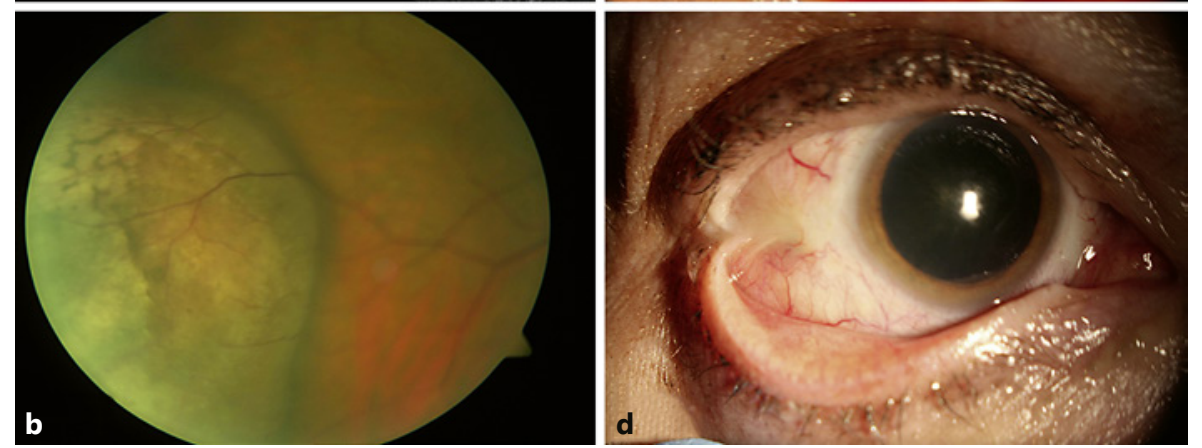

and scleritis resolved, she did develop a tight symblepharon in that area (Fig. 3d). A year after the initial surgery, she underwent symblepharon repair with placement of amniotic membrane and concurrent cataract surgery. At the last follow-up, 43 months from the date of the plaque placement, she had scarring and residual symblepharon but no progression of scleral thinning or necrosis was noted.

\section{Comment}

We present three patients who had varying degrees of early post-iodine-125 plaque brachytherapy conjunctival dehiscence and scleral necrosis. A review of the literature reveals that in nearly all reported cases of scleral 
necrosis following plaque brachytherapy, the interval between treatment and development of the scleral defect has been several months to several years. We found only a single case report that occurred before postoperative month $4[5,6]$. While corneoscleral necrosis as a complication of plaque brachytherapy has been reported in the literature, we believe our cases, presenting sooner in the postoperative course, represent a distinct clinical entity [3, 6-9].

Although sclera is relatively radioresistant, there are reports of scleral changes after radiotherapy. Gündüz and colleagues [9] showed that radiation-induced scleral necrosis was found in 5\% of cases at 1 year, increasing to $12 \%$ at 5 years after plaque radiotherapy for ciliary body melanoma. The patients in the aforementioned study were treated with a variety of different radioisotopes (iodine-125 in 45\%, cobalt 60 in $45 \%$, ruthenium-106 in 9\%, and iridium-192 in 0.7\%). This is in contrast to an incidence of $1 \%$ in all uveal melanomas treated with iodine-125 brachytherapy in a large series of 4,831 eyes with the necrosis developing between 5 and 351 months following the brachytherapy [7]. It is noteworthy that all of the cases reviewed by Gündüz et al. [9] were primary ciliary body tumors as this more anterior location may explain the increased incidence of scleral necrosis observed in this cohort. It has been reported that most cases of radiation-induced scleral necrosis were observed while $14 \%$ underwent a scleral patch graft and $4 \%$ progressed to a full-thickness perforation [7]. Management options described include observation, artificial lubrication, tissue glue, conjunctival graft/flap, amniotic membrane transplantation, scleral patch graft, dermal patch graft, and Tenon's fascia transposition. Factors predictive of scleral necrosis included tumor thickness $\geq 6 \mathrm{~mm}$, ciliary body and peripheral choroidal location, and radiation dose to sclera $\geq 400$ Gy to the outer sclera. Two of the three cases in our series had an anterior location of the tumor but all were $<6 \mathrm{~mm}$ in height.

A common feature of all three cases is that conjunctival dehiscence and scleral necrosis occurred near the site of muscle disinsertion and reattachment. Surgically-induced necrotizing scleritis (SINS) is a destructive form of scleritis that can occur at the site of surgical trauma to the sclera [10]. SINS has not been previously reported following plaque brachytherapy but is a rare complication following any ocular surgery, including strabismus surgery involving the extraocular muscles [11]. In our cases, surgical trauma to the sclera from the transscleral biopsy and/or muscle disinsertion and reattachment could have

Conjunctival Dehiscence after Plaque

Brachytherapy resulted in SINS. Specifically, given the spatial relationship between the conjunctival dehiscence and scleral necrosis and the operated muscle(s) in our cases, this should be considered as a possible etiology.

SINS typically features aggressive necrotizing scleritis requiring treatment with systemic corticosteroids and/or immunosuppressive therapy $[10,12]$. Few cases of SINS following extraocular muscle surgery have been reported with a presentation time ranging from 5 days to 51 years following surgery. In a large review, 9 of 43 patients developed SINS at the site of previous muscle surgery. Of these, 5 had a mean onset of 21.7 years after a single procedure, while the remaining 4 had a history of multiple surgeries and a mean onset time of 10 weeks [10].

Several possible mechanisms may explain the early development of conjunctival dehiscence and scleral necrosis in these patients. First, a direct necrotizing effect from the radiation itself could lead to poor wound healing and subsequent dehiscence; however, the radiation doses delivered to these patients was not significantly higher than typical doses and the gold covering from the COMS plaque would shield the overlying conjunctiva, but not the scleral base. Second, given the association between the location of conjunctival dehiscence and scleral necrosis and the operated muscles, SINS is a possible etiology though none of the three patients had any significant past medical or ocular history suggestive of an occult systemic autoimmune phenomenon nor had any undergone prior ocular surgery making SINS unlikely. Moreover, the clinical course was mild compared to that which is typical of SINS and all responded well to topical treatment, which is in contrast to SINS, which usually requires aggressive systemic immunosuppressive therapies. A third possibility is that local mechanical trauma and inflammation from the transscleral biopsy and the brachytherapy procedure itself could have resulted in poor wound healing. Fourth, these eyes may have had an indirect effect secondary to inflammation related to rapid tumor necrosis following plaque therapy. Finally, given the acuity of these cases, a conjunctival microinfection, as seen within the setting of an exposed scleral buckle, is possible [13]. While no cultures or other microbiological studies were undertaken at the time of presentation, all eyes responded well to a combination of topical antibiotics and steroids and did not require repeat surgery to close the tissue defect. It is unlikely that this was due to poor wound closure as all conjunctival sutures were intact on postoperative day and week 1. 
Although radiation- or surgery-induced scleral thinning and necrosis are well-known entities, there is a paucity of literature indicating limits of scleral tolerability to the effects of brachytherapy treatments. Animal studies of rabbit eyes showed early disruption of the scleral lamellae at even a low dose of $1 \mathrm{~Gy}$ and loss of scleral cells at 5 Gy [14]. It is possible that preoperative scleral imaging with ultrasound biomicroscopy and/or anterior segment optical coherence tomography could help detect eyes with thin sclera that might be more predisposed to scleral necrosis.

While the exact cause of the conjunctival dehiscence and scleral necrosis in these patients cannot be identified, this remains an exceedingly rare and likely underreported complication. However, it is important to recognize that this can occur early in the postoperative course and conservative management is often successful.

\section{Acknowledgement}

This work was partially supported by the NIH Core Grant for Vision Research EY05722 and the Unrestricted RPB Grant from Research to Prevent Blindness Inc. (both to Duke University Department of Ophthalmology).

\section{Statement of Ethics}

The research was performed in adherence to the tenets of the Declaration of Helsinki. The report of this case series was determined not to require institutional review board (IRB) review and approval by the Duke University Hospital IRB.

\section{Disclosure Statement}

None of the authors have any sponsorship or funding arrangements relating to this research.

\section{References}

1 Jones IS, Reese AB: Focal scleral necrosis; a late sequel of irradiation. AMA Arch Ophthalmol 1953;49:633-636.

2 Parsons JT, Bova FJ, Mendenhall WM, et al: Response of the normal eye to high dose radiotherapy. Oncology (Williston Park) 1996; 10:837-847; discussion 847-848, 851-852.

3 Macfaul PA and Bedford MA. Ocular complications after therapeutic irradiation. Br J Ophthalmol 1970;54:237-247.

4 Rivard MJ, Coursey BM, DeWerd LA, et al: Update of AAPM Task Group No. 43 Report: A revised AAPM protocol for brachytherapy dose calculations. Med Phys 2004;31:633674.

5 Correa ZM, Augsburger JJ, Freire J, Eagle RC Jr: Early-onset scleral necrosis after iodine I 125 plaque radiotherapy for ciliochoroidal melanoma. Arch Ophthalmol 1999;117:259261.
6 Chaudhry IA, Liu M, Shamsi FA, et al: Corneoscleral necrosis after episcleral Au-198 brachytherapy of uveal melanoma. Retina 2009;29:73-79.

7 Kaliki S, Shields CL, Rojanaporn D, et al: Scleral necrosis after plaque radiotherapy of uveal melanoma: a case-control study. Ophthalmology 2013;120:1004-1011.

8 Radin PP, Lumbroso-Le Rouic L, Levy-Gabriel C, et al: Scleral necrosis after radiation therapy for uveal melanomas: report of 23 cases. Graefes Arch Clin Exp Ophthalmol 2008;246: 1731-1736.

9 Gündüz K, Shields CL, Shields JA, et al: Plaque radiotherapy of uveal melanoma with predominant ciliary body involvement. Arch Ophthalmol 1999;117:170-177.
10 O'Donoghue E, Lightman S, Tuft S, Watson P: Surgically induced necrotising sclerokeratitis (SINS) - precipitating factors and response to treatment. Br J Ophthalmol 1992; 76:17-21.

11 Bradbury JA, Taylor RH: Severe complications of strabismus surgery. J AAPOS 2013; 17:59-63.

12 Sainz de la Maza M, Foster CS: Necrotizing scleritis after ocular surgery. A clinicopathologic study. Ophthalmology 1991;98:17201726.

13 Smiddy WE, Miller D, Flynn HW Jr: Scleral buckle removal following retinal reattachment surgery: clinical and microbiologic aspects. Ophthalmic Surg 1993;24:440-445.

14 Kunz J, Lommatzsch P: Changes of the S35 sulfate incorporation into the sclera of rabbit eyes following high-dosed beta irradiation (in German). Graefes Arch Clin Exp Ophthalmol 1966;171:68-76. 